\title{
Desempenho de cordeiros Santa Inês do nascimento ao desmame filhos de ovelhas alimentadas com diferentes níveis de energia ${ }^{1}$
}

\section{Performance from birth to weaning of Santa Inês lambs born to ewes fed different levels of energy}

\author{
Filipe Alexandre Boscaro de Castro $^{2 *}$; Edson Luis de Azambuja Ribeiro ${ }^{3}$; \\ Natália Albieri Koritiaki ${ }^{4}$; Ivone Yurika Mizubuti³; \\ Leandro das Dores Ferreira da Silva²; Elzânia Sales Pereira ${ }^{5}$; \\ Andréa Pereira Pinto ${ }^{5}$; Camila Constantino ${ }^{4}$; Francisco Fernandes Junior ${ }^{6}$
}

\section{Resumo}

O principal objetivo deste trabalho foi avaliar a influência dos níveis de energia na alimentação de ovelhas no terço final da gestação e lactação sobre os pesos e medidas corporais de cordeiros do nascimento ao desmame. Como objetivos secundários avaliaram-se também os efeitos do ano de nascimento, do sexo da cria, do tipo de nascimento e da idade da ovelha ao parto sobre as mesmas características. Foram avaliados 45 cordeiros da raça Santa Inês, filhos de 40 ovelhas distribuídas de forma inteiramente casualizada em três tratamentos que se diferenciaram quanto ao nível energético da dieta $(2,0 ; 2,2$ e 2,4 Mcal de EM/kg de MS). As ovelhas estavam sujeitas as dietas experimentais durante o terço final da gestação e lactação. Os cordeiros permaneceram com suas mães até o desmame, realizado aos 70 dias. O nível de energia da alimentação das ovelhas não afetou os parâmetros mensurados nos cordeiros ao nascimento. A análise de regressão mostrou efeito linear crescente dos níveis de energia sobre todas as características avaliadas no desmame e nos ganhos até o desmame. Cordeiros machos apresentaram maiores pesos ao nascimento e ao desmame. Todas as medidas morfométricas realizadas foram semelhantes entre machos e fêmeas, com exceção para perímetro torácico aos 70 dias, que foi maior em cordeiros machos. Os cordeiros nascidos de partos simples foram maiores e mais pesados. Cordeiros filhos de ovelhas com idade intermediária (4 e 6 dentes) obtiveram maiores médias para ganho médio diário de peso. O perímetro torácico foi a medida que melhor se correlacionou com o peso, tanto ao nascimento como ao desmame. Além da nutrição da ovelha é importante considerar outros fatores ambientais que afetam as características de crescimento dos animais por atuarem na expressão do seu potencial genético.

Palavras-chave: Fatores ambientais, medidas corporais, ovinos, restrição energética, seleção

\footnotetext{
${ }^{1}$ Parte da tese de Doutorado em Ciência Animal, do primeiro autor, Universidade Estadual de Londrina, UEL, Londrina, PR.

${ }^{2}$ Profs. Drs. do Dept ${ }^{\circ}$ de Zootecnia, Universidade Estadual de Londrina, UEL, Cx. Postal 6001, 86001-970. Londrina, PR. E-mail: fabcastro76@yahoo.com.br; leandro@uel.br

${ }^{3}$ Profs. Drs. do Dept ${ }^{\mathrm{o}}$ de Zootecnia, UEL, Bolsista de Produtividade do CNPq. Londrina, PR. E-mail: elar@uel.br; mizubuti@uel. br

${ }^{4}$ Discente(s) de Doutorado do Programa de Pós-Graduação em Ciência Animal, UEL, Londrina, PR. E-mail: nataliaalbieri@ hotmail.com; caconstantino@hotmail.com

${ }_{5}^{5}$ Profs. Drs. do Dept ${ }^{\mathrm{o}}$ de Zootecnia, Universidade Federal do Ceará, , UFC, Ceará, CE. Bolsista de Produtividade do CNPq. E-mail: elzania@hotmail.com; deiapp@hotmail.com

${ }^{6}$ Discente de Mestrado do Programa de Pós-Graduação em Ciência Animal, UEL, Londrina, PR. E-mail: ffjunior_zoo@hotmail. com

* Autor para correspondência
} 


\begin{abstract}
The main objective of this study was to evaluate the influence of the energy levels in the diet of ewes in the last third of gestation and lactation on body measurements and weights of lambs from birth to weaning. As secondary objectives were also evaluated the effects of year of birth, sex of the lamb, type of birth and age of the ewe at lambing on the same characteristics. Forty-five Santa Inês lambs were evaluated. They were born from 40 ewes distributed randomly to three treatments: 2.0, 2.2 and 2.4 Mcal of metabolizable energy $/ \mathrm{kg}$ of dry matter). Ewes were subjected to the experimental diets during the last third of gestation and lactation. Lambs were kept with their mothers until weaning, which occurred at 70 days. Feeding energy level did not affect traits measured on the lambs at birth. Regression analysis showed a linear increasing in all traits at weaning and in average daily gain until weaning as the energy level increased. Male lambs were heavier at birth and at weaning. All morphometric measurements performed were similar between males and females, except for thoracic perimeter at 70 days, which was higher in male lambs. Single lambs were larger and heavier than twin lambs. Lambs born from ewes of intermediate age (4 and 6 teeth) showed higher means for average daily weight gain. Among morphometric measurements, thoracic perimeter showed the best correlation to body weight at birth and at weaning. Besides the nutrition of the ewe, it is important to consider other environmental factors that affect animal's growth characteristics by acting on the expression of genetic potential.
\end{abstract}

Key words: Body measurements, energy restriction, environmental factors, selection, sheep

\section{Introdução}

A criação de ovinos para produção de carne tem apresentado incremento em todas as regiões do Brasil, e vem se tornando uma atividade econômica importante. Entretanto, para que esta seja viável é necessário o controle dos efeitos ambientais e genéticos que afetam o desenvolvimento dos animais, bem como a correta utilização dos manejos nutricionais, reprodutivos e sanitários.

A raça Santa Inês está difundida em diversas regiões do país, principalmente no Nordeste. É uma raça de grande importância devido a sua rusticidade, prolificidade, menor exigência nutricional, menor estacionalidade reprodutiva, acentuada habilidade materna e a sua resistência e capacidade de adaptação às condições adversas de clima (temperaturas elevadas). Contudo é um material genético novo do ponto de vista biológico, e de certa forma ainda pouco conhecido (CARNEIRO et al., 2007).

A velocidade de crescimento dos cordeiros é um aspecto de extrema importância para produção de carne ovina, pois quanto mais cedo e com menor custo atingirem as condições de abate, maior será o impacto positivo para o sistema de produção. Portanto torna-se necessário o conhecimento dos componentes que influenciam o desenvolvimento dos animais e, consequentemente, a produção (PACHECO; QUIRINO, 2008).

De acordo com Geraseev et al. (2006), a nutrição inadequada da ovelha, particularmente no estágio final da gestação, pode comprometer o desenvolvimento de alguns órgãos do feto alterando a fisiologia do animal. Este fato pode resultar em modificações no crescimento pré e pós-natal, além da idade e do peso em que ocorre a aceleração ou desaceleração do crescimento de cada tecido, resultando em alteração na composição corporal desses animais.

O controle zootécnico é fundamental para o sucesso da ovinocultura, sendo os dados sobre o desempenho ponderal uma importante ferramenta para realizá-lo. A falta de controle do peso por meio da utilização de balança é a realidade de muitos produtores, devido ao alto custo e da falta de mobilidade do equipamento. Nesse contexto as medidas corporais constituem uma alternativa viável, pois por meio delas é possível prever o peso corporal devida à alta correlação existente entre essas características (SOUZA et al., 2009). Além disso, medidas como o comprimento do corpo, perímetro torácico e altura de cernelha são 
importantes indicadores do rendimento da carcaça e da capacidade digestiva e respiratória dos animais (SANTANA; COSTA; FONSECA, 2001).

O principal objetivo deste trabalho foi avaliar a influência dos níveis de energia da alimentação de ovelhas no terço final da gestação e durante a lactação sobre os pesos e características morfométricas dos cordeiros no período pré-desmame; e como objetivos secundários, avaliar os efeitos do ano de nascimento, do sexo da cria, do tipo de nascimento (simples ou gemelar) e da idade da ovelha ao parto sobre as mesmas características de desempenho.

\section{Material e Métodos}

O trabalho foi conduzido no setor de ovinocultura da Fazenda Escola da Universidade Estadual de Londrina (UEL) de Setembro a Dezembro de 2009 e de Junho a Setembro de 2010. Esta propriedade localiza-se no município de Londrina (Lat. S. 2320'10" e Long. W. 5109'15"), com 610 metros de altitude, temperatura ambiente média anual variando de $21,0^{\circ} \mathrm{C}$ a $22,0^{\circ} \mathrm{C}$, sendo a média dos meses mais quentes (Janeiro e Fevereiro) de $24,5^{\circ} \mathrm{C}$ e a média do mês mais frio (Junho) $16,5^{\circ} \mathrm{C}$. A precipitação média anual varia de 1400 a $1600 \mathrm{~mm}$, sendo Dezembro, Janeiro e Fevereiro os meses mais chuvosos e Junho, Julho e Agosto os meses mais secos (CAVIGLIONE et al., 2000).

Foram utilizadas 40 ovelhas em idade adulta com média de 105 dias de gestação no início do experimento, sendo avaliados os 45 cordeiros da raça Santa Inês provenientes dos partos destas fêmeas. As ovelhas foram confinadas em baias individuais em aprisco coberto e com piso ripado, distribuídas de forma inteiramente casualizada em três tratamentos que se diferenciaram quanto ao nível energético $(2,0 ; 2,2$ e 2,4 Mcal de energia metabolizável $(\mathrm{EM}) / \mathrm{kg}$ de matéria seca (MS)) da dieta (Tabela 1). O nível 2,4 correspondeu à exigência energética para o terço final da gestação de ovelhas de $60 \mathrm{~kg}$ (NRC, 2007). Os outros níveis corresponderam a restrições em energia.

Tabela 1. Composição bromatológica das rações experimentais (\%MS).

\begin{tabular}{lccc}
\hline \multirow{2}{*}{ Componentes nutritivos } & \multicolumn{3}{c}{ Nível de energia metabolizável (Mcal/kg de MS) } \\
\cline { 2 - 4 } & $\mathbf{2 , 4}$ & $\mathbf{2 , 2}$ & $\mathbf{2 , 0}$ \\
\hline Matéria seca & 35,91 & 31,24 & 28,52 \\
Matéria orgânica & 93,09 & 92,75 & 92,21 \\
Proteína bruta & 10,00 & 10,20 & 10,45 \\
Extrato etéreo & 1,95 & 1,68 & 1,26 \\
Matéria mineral & 6,91 & 7,25 & 7,79 \\
Fibra em detergente neutro & 51,30 & 63,70 & 72,95 \\
Fibra em detergente ácido & 28,00 & 35,30 & 40,65 \\
Nutrientes digestíveis totais ${ }^{1}$ & 65,75 & 60,00 & 55,45 \\
\hline
\end{tabular}

${ }^{1}$ Estimado a partir da composição dos alimentos segundo equações propostas por McDowell et al. (1974).

Fonte: Elaboração dos autores.

As rações experimentais consistiram de silagem de sorgo e ração concentrada composta por farelo de milho, farelo de soja, uréia, calcário calcítico, fosfato bicálcico e sal mineral em proporções variando em função do nível energético (Tabela 2).
A alimentação das ovelhas foi fornecida a vontade durante o terço final da gestação e lactação. Os cordeiros permaneceram com suas mães, tendo acesso à alimentação das mesmas e foram desmamados com 70 dias de idade. 
Para as análises dos efeitos foram utilizadas as características de peso e medidas corporais realizadas ao nascimento e ao desmame. As mensurações de morfometria corporal foram realizadas com o auxílio de fita métrica, com o animal mantido em posição correta de aprumos. A altura da cernelha foi medida entre o ponto mais alto da região interescapular e o solo. O comprimento corporal foi medido da cernelha até a parte caudal da tuberosidade isquiática. O perímetro torácico foi medido na circunferência externa da cavidade torácica, junto às axilas.

Tabela 2. Composição das rações experimentais (\%MS).

\begin{tabular}{lrrr}
\hline \multirow{2}{*}{ Componentes nutritivos } & \multicolumn{3}{c}{ Nível de energia metabolizável (Mcal/kg de MS) $^{2}$} \\
\cline { 2 - 4 } & $\mathbf{2 , 4}$ & $\mathbf{2 , 2}$ & $\mathbf{2 , 0}$ \\
\hline Silagem de sorgo & 63,2 & 82,2 & 96,5 \\
Farelo de milho & 32,6 & 13,1 & 0,0 \\
Farelo de soja & 1,9 & 2,7 & 1,3 \\
Uréia & 0,5 & 0,5 & 0,7 \\
Fosfato bicálcico & 0,3 & 0,3 & 0,4 \\
Calcário calcitico $_{\text {Sal mineral }^{1}}$ & 0,5 & 0,2 & 0,1 \\
\hline
\end{tabular}

${ }^{1}$ Níveis de garantia do fabricante para cada 1000g: cálcio (mínimo), 128g; cálcio (máximo), 155g; sódio, 152mg; fósforo, 60g; enxofre, 10g; magnésio, 6g; cobalto, 50mg; ferro, 1400mg; iodo, 74mg; manganês, 1820mg; selênio, 15mg; zinco, 2730mg; flúor (máximo), 600mg.

Fonte: Elaboração dos autores.

As características estudadas foram submetidas à análise de variância, tendo como variáveis independentes o nível de energia da alimentação da ovelha (2,0; 2,2 ou 2,4 Mcal de EM/kg de MS), o tipo de nascimento (simples ou gemelar), o sexo (macho ou fêmea), a idade da mãe ao parto $(2,4$, 6 ou 8 dentes incisivos permanentes) e o ano de nascimento do cordeiro (2009 e 2010). As interações entre as variáveis foram desconsideradas na análise final por não apresentarem efeito significativo $(\mathrm{P}>0,10)$. As médias do nível de energia da alimentação da ovelha e da idade da mãe ao parto foram comparadas pelo teste Tukey e as demais, pelo teste F. Quando significativos, o efeito do nível de energia foi submetido à análise de regressão. Todas as análises estatísticas foram realizadas com auxílio do programa estatístico SAS (versão 8.2).

\section{Resultados e Discussão}

O nível de energia da alimentação das ovelhas no terço final de gestação não afetou os parâmetros mensurados nos cordeiros ao nascimento (Tabela 3 ), apresentando médias de $3,60 \mathrm{~kg}, 37,28 \mathrm{~cm}$, $26,74 \mathrm{~cm}$ e $39,45 \mathrm{~cm}$ para peso, perímetro torácico, comprimento corporal e altura de cernelha, respectivamente.

A média de peso ao nascer foi similar aos 3,61; 3,63 e $3,77 \mathrm{~kg}$ observados, respectivamente, por Sarmento et al. (2006), Fernandes et al. (2007) e Ribeiro et al. (2008a), em ovinos Santa Inês.

Castro et al. (2012), trabalhando com os mesmos níveis energéticos na alimentação das ovelhas no terço final da gestação observaram efeito linear crescente das dietas sobre o peso ao nascer de cordeiros.

De acordo com Gerassev et al. (2006) e Castro et al. (2012), o peso ao nascimento de cordeiros é uma característica determinante na sobrevivência neonatal e no desempenho futuro dos animais, sendo um indicativo da eficiência produtiva do rebanho.

Entretanto, Rae et al. (2001) afirmaram que 
a nutrição inadequada na fase pré-natal não está necessariamente associada ao baixo peso ao nascimento e nem tem, obrigatoriamente, consequências negativas nas primeiras semanas de vida. Alguns efeitos podem ser evidenciados em estágios mais avançados na vida do animal (MARTIN; RODGER; BLACHE, 2004). Tais prejuízos estão relacionados com a diferenciação e desenvolvimento do sistema reprodutivo (RAE et al., 2001) e com a formação das fibras musculares (GREENWOOD et al., 2000), características determinantes da eficiência reprodutiva e da qualidade da carcaça, respectivamente.

Tabela 3. Médias e erros-padrão do peso, perímetro torácico, comprimento e altura ao nascimento e ao desmame e ganhos médios diários do nascimento ao desmame dessas características de acordo com o nível de energia da alimentação da ovelha e o ano de nascimento.

\begin{tabular}{|c|c|c|c|c|c|}
\hline \multirow{2}{*}{ Variáveis } & \multicolumn{3}{|c|}{ Nível de Energia Metabolizável (Mcal / kg de MS) } & \multicolumn{2}{|c|}{ Ano } \\
\hline & 2,0 & 2,2 & 2,4 & 2009 & 2010 \\
\hline Peso ao nascimento, $\mathrm{kg}$ & $3,52 \pm 0,24$ & $3,67 \pm 0,18$ & $3,61 \pm 0,19$ & $3,69 \pm 0,15$ & $3,52 \pm 0,22$ \\
\hline Peso ao desmame $(\mathrm{P} 70)^{1}, \mathrm{~kg}$ & $11,04 \pm 0,93^{\mathrm{b}}$ & $11,98 \pm 0,69^{\mathrm{ab}}$ & $13,24 \pm 0,73^{\mathrm{a}}$ & $11,70 \pm 0,59$ & $12,47 \pm 0,84$ \\
\hline $\begin{array}{l}\text { Ganho médio diário de peso } \\
(\mathrm{GMP})^{2}, \mathrm{~kg}\end{array}$ & $0,107 \pm 0,012^{b}$ & $0,119 \pm 0,009^{\mathrm{ab}}$ & $0,138 \pm 0,010^{\mathrm{a}}$ & $0,114 \pm 0,008$ & $0,128 \pm 0,011$ \\
\hline $\begin{array}{l}\text { Perímetro Torácico ao } \\
\text { nascimento, } \mathrm{cm}\end{array}$ & $36,20 \pm 1,04$ & $38,21 \pm 0,76$ & $37,44 \pm 0,81$ & $37,49 \pm 0,66$ & $37,08 \pm 0,94$ \\
\hline $\begin{array}{l}\text { Perímetro Torácico ao } \\
\text { desmame }(\mathrm{PT} 70)^{3}, \mathrm{~cm}\end{array}$ & $53,49 \pm 1,32^{b}$ & $54,59 \pm 0,98^{b}$ & $57,01 \pm 1,03^{\mathrm{a}}$ & $53,86 \pm 0,84^{b}$ & $56,20 \pm 1,20^{\mathrm{a}}$ \\
\hline $\begin{array}{l}\text { Ganho médio diário de } \\
\text { perímetro }(\mathrm{GMPT})^{4}, \mathrm{~cm}\end{array}$ & $0,247 \pm 0,021^{\mathrm{ab}}$ & $0,234 \pm 0,016^{\mathrm{b}}$ & $0,279 \pm 0,017^{\mathrm{a}}$ & $0,234 \pm 0,014^{b}$ & $0,273 \pm 0,019^{a}$ \\
\hline $\begin{array}{l}\text { Comprimento ao } \\
\text { nascimento, } \mathrm{cm}\end{array}$ & $25,99 \pm 0,61$ & $27,18 \pm 0,45$ & $27,04 \pm 0,48$ & $26,95 \pm 0,39$ & $26,53 \pm 0,55$ \\
\hline $\begin{array}{l}\text { Comprimento ao desmame } \\
(\mathrm{C} 70)^{5}, \mathrm{~cm}\end{array}$ & $40,88 \pm 1,07^{\mathrm{c}}$ & $42,59 \pm 0,79^{b}$ & $44,80 \pm 0,84^{\mathrm{a}}$ & $41,76 \pm 0,68^{b}$ & $43,75 \pm 0,97^{\mathrm{a}}$ \\
\hline $\begin{array}{l}\text { Ganho médio diário de } \\
\text { comprimento }(\mathrm{GMC})^{6}, \mathrm{~cm}\end{array}$ & $0,213 \pm 0,017^{\mathrm{b}}$ & $0,220 \pm 0,013^{b}$ & $0,254 \pm 0,014^{\mathrm{a}}$ & $0,212 \pm 0,011^{\mathrm{b}}$ & $0,246 \pm 0,016^{\mathrm{a}}$ \\
\hline Altura ao nascimento, $\mathrm{cm}$ & $39,41 \pm 0,81$ & $39,37 \pm 0,60$ & $39,58 \pm 0,64$ & $38,94 \pm 0,52$ & $49,97 \pm 0,74$ \\
\hline $\begin{array}{l}\text { Altura ao desmame }(\mathrm{A} 70)^{7} \text {, } \\
\mathrm{cm}\end{array}$ & $51,30 \pm 1,51^{\mathrm{b}}$ & $54,68 \pm 1,11^{\mathrm{ab}}$ & $55,75 \pm 1,18^{a}$ & $53,92 \pm 0,96$ & $53,90 \pm 1,34$ \\
\hline $\begin{array}{l}\text { Ganho médio diário de } \\
\text { altura }(\mathrm{GMA})^{8}, \mathrm{~cm}\end{array}$ & $0,170 \pm 0,021^{\mathrm{b}}$ & $0,219 \pm 0,015^{\mathrm{a}}$ & $0,231 \pm 0,016^{\mathrm{a}}$ & $0,214 \pm 0,013$ & $0,199 \pm 0,019$ \\
\hline
\end{tabular}

$\mathrm{a}, \mathrm{b}=$ médias seguidas de letras diferentes na coluna diferem significativamente $(\mathrm{P}<0,10)$ entre si.

$1,2,3,4,5,6,7,8=$ equações de regressão, onde $\mathrm{X}$ representa o nível de energia da dieta.

${ }^{1} \mathrm{P} 70=-2,5497+7,4434 \mathrm{X}, \mathrm{R}^{2}=0,99$;

${ }^{2} \mathrm{GMP}=-0,0782+0,0994 \mathrm{X}, \mathrm{R}^{2}=0,97 ;$

${ }^{3} \mathrm{PT} 70=34,48+10,21 \mathrm{X}, \mathrm{R}^{2}=0,94$;

${ }^{4} \mathrm{GMPT}=0,0172+0,1130 \mathrm{X}, \mathrm{R}^{2}=0,42$;

${ }^{5} \mathrm{C} 70=22,40+10,00 \mathrm{X}, \mathrm{R}^{2}=0,99 ;$

${ }^{6} \mathrm{GMC}=-0,0126+0,1167 \mathrm{X}, \mathrm{R}^{2}=0,86 ;$

${ }^{7} \mathrm{~A} 70=34,08+10,16 \mathrm{X}, \mathrm{R}^{2}=0,99 ;$

${ }^{8} \mathrm{GMA}=-0,0378+0,1243 \mathrm{X}, \mathrm{R}^{2}=0,87$.

Fonte: Elaboração dos autores. 
Já as características avaliadas ao desmame (70 dias de idade) e os ganhos médios diários até o desmame sofreram influência das dietas experimentais (Tabela 3). Essas médias apresentaram-se mais elevadas nos cordeiros filhos de ovelhas que receberam a dieta com maior teor de energia (2,4 Mcal de EM/Kg de MS), e menores no tratamento menos energético (2,0 Mcal de EM/Kg de MS). Estes resultados são confirmados pela análise de regressão, que mostrou efeito linear crescente dos níveis de energia sobre as características avaliadas no desmame e nos ganhos até o desmame (Tabela 3), comprovando o comprometimento no desenvolvimento dos filhos de ovelhas submetidas à restrição energética pré e pós-natal.

O peso do cordeiro ao desmame sofre influência direta da produção de leite da mãe (MOHAMMADI et al., 2010) que, por sua vez, é afetada pelo nível energético da alimentação da matriz (CASTRO et al., 2012).

Os valores médios para peso ao desmame dos animais $(11,04,11,98$ e 13,24 $\mathrm{kg}$ para os grupos 2,0; 2,2 e 2,4, respectivamente) foram inferiores ao 14,76 $\mathrm{kg}$ relatado por Gerassev et al. (2006). Cordeiros das dietas contendo 2,0 e 2,2 Mcal de EM/kg de MS apresentaram, respectivamente, ganhos médios diários de peso 22,5\% e 13,8\% inferiores à dieta contendo 2,4 Mcal de EM/kg de MS.

Mesmo no grupo de maior nível energético, os cordeiros apresentaram ganhos de peso inferiores ao observado por Geraseev et al. (2006) para a raça Santa Inês $(0,188 \mathrm{~kg} / \mathrm{dia})$ e ao observado por Carvalho et al. (1999) em cordeiros $3 / 4$ Texel $(0,316$ $\mathrm{kg} / \mathrm{dia})$.

Mexia et al. (2004) destacaram o ganho de peso dos cordeiros como importante parâmetro do desempenho produtivo. De acordo com os autores, além de sofrer influência da nutrição materna, a velocidade de crescimento pode estar sob efeito do sexo do animal e do tipo de parto (simples ou gemelar).

$\mathrm{O}$ ano de nascimento não afetou as medidas dos cordeiros ao nascer, no entanto afetou o perímetro torácico e o comprimento ao desmame e os ganhos médios dessas características até o desmame, onde os animais nascidos no ano de 2009 apresentaram médias inferiores (Tabela 3). De acordo com Sousa et al. (2006) as diferenças no desenvolvimento dos animais observadas entre os vários anos são atribuídas aos fatores climáticos, como precipitação, temperatura e umidade, que acarretam efeitos diferenciados tanto nos animais quanto na quantidade e na qualidade do alimento disponibilizado. Além desses fatores, destacam-se as mudanças no manejo e alterações na composição genética do rebanho.

Com relação ao sexo do cordeiro, os machos apresentaram maiores pesos ao nascimento e ao desmame (Tabela 4). Esses resultados concordam com os obtidos por Fernandes, Buchanan e SelaiveVillarroel (2001), por Barros, Vasconcelos e Lobo (2004) e por Mohammadi et al. (2010). No presente trabalho, cordeiros machos foram $0,43 \mathrm{~kg}$ mais pesados ao nascimento e $1,81 \mathrm{~kg}$ ao desmame. De acordo com Pacheco e Quirino (2008) e Mohammadi et al. (2010), a diferença entre os sexos ocorre principalmente devido a diferenças nos cromossomos sexuais, provavelmente na posição dos genes relacionados ao crescimento, e devido a diferenças no sistema endócrino, especialmente nos hormônios sexuais. 
Tabela 4. Médias e erros-padrão do peso, perímetro torácico, comprimento e altura ao nascimento e ao desmame e ganhos médios diários do nascimento ao desmame dessas características de acordo com o sexo do cordeiro e o tipo de nascimento.

\begin{tabular}{lcccc}
\hline \multirow{2}{*}{ Variáveis } & \multicolumn{2}{c}{ Sexo } & \multicolumn{2}{c}{ Tipo de Nascimento } \\
\cline { 2 - 5 } & Fêmea & Macho & Simples & Gemelar \\
\hline Peso ao nascimento, $\mathrm{kg}$ & $3,39 \pm 0,21^{\mathrm{b}}$ & $3,82 \pm 0,15^{\mathrm{a}}$ & $4,10 \pm 0,12^{\mathrm{a}}$ & $3,10 \pm 0,25^{\mathrm{b}}$ \\
Peso ao desmame, $\mathrm{kg}$ & $11,18 \pm 0,79^{\mathrm{b}}$ & $12,99 \pm 0,56^{\mathrm{a}}$ & $15,16 \pm 0,47^{\mathrm{a}}$ & $9,01 \pm 0,97^{\mathrm{b}}$ \\
Ganho médio diário de peso, $\mathrm{kg}$ & $0,111 \pm 0,011$ & $0,131 \pm 0,007$ & $0,158 \pm 0,006^{\mathrm{a}}$ & $0,084 \pm 0,013^{\mathrm{b}}$ \\
Perímetro Torácico ao nascimento, cm & $36,97 \pm 0,89$ & $37,59 \pm 0,63$ & $38,76 \pm 0,52^{\mathrm{a}}$ & $35,81 \pm 1,09^{\mathrm{b}}$ \\
Perímetro Torácico ao desmame, cm & $53,92 \pm 1,12^{\mathrm{b}}$ & $56,14 \pm 0,79^{\mathrm{a}}$ & $59,17 \pm 0,67^{\mathrm{a}}$ & $50,89 \pm 1,38^{\mathrm{b}}$ \\
Ganho médio diário de perímetro, cm & $0,242 \pm 0,018$ & $0,265 \pm 0,013$ & $0,292 \pm 0,011^{\mathrm{a}}$ & $0,216 \pm 0,022^{\mathrm{b}}$ \\
Comprimento ao nascimento, cm & $26,52 \pm 0,52$ & $29,95 \pm 0,37$ & $27,66 \pm 0,31^{\mathrm{a}}$ & $25,82 \pm 0,64^{\mathrm{b}}$ \\
Comprimento ao desmame, cm & $42,17 \pm 0,91$ & $43,34 \pm 0,65$ & $45,68 \pm 0,54^{\mathrm{a}}$ & $39,82 \pm 1,12^{\mathrm{b}}$ \\
Ganho médio diário de comprimento, cm & $0,224 \pm 0,019$ & $0,234 \pm 0,010$ & $0,258 \pm 0,009^{\mathrm{a}}$ & $0,200 \pm 0,018^{\mathrm{b}}$ \\
Altura ao nascimento, cm & $39,04 \pm 0,69$ & $39,87 \pm 0,49$ & $40,42 \pm 0,41^{\mathrm{a}}$ & $38,48 \pm 0,85^{\mathrm{b}}$ \\
Altura ao desmame, cm & $53,03 \pm 1,29$ & $54,78 \pm 0,91$ & $57,20 \pm 0,76^{\mathrm{a}}$ & $50,61 \pm 1,57^{\mathrm{b}}$ \\
Ganho médio diário de altura, cm & $0,200 \pm 0,018$ & $0,213 \pm 0,012$ & $0,240 \pm 0,010^{\mathrm{a}}$ & $0,173 \pm 0,021^{\mathrm{b}}$ \\
\hline
\end{tabular}

$\mathrm{a}, \mathrm{b}=$ médias seguidas de letras diferentes na coluna diferem significativamente $(\mathrm{P}<0,10)$ entre si.

Fonte: Elaboração dos autores.

O ganho médio diário de peso do nascimento ao desmame observado neste trabalho foi semelhante para machos e fêmeas.

Todas as medidas morfométricas realizadas foram semelhantes entre machos e fêmeas, com exceção para perímetro torácico aos 70 dias, que foi maior em cordeiros machos.

O tipo de nascimento afetou todas as características avaliadas, sendo que os cordeiros nascidos de partos simples foram maiores e mais pesados em relação aos nascidos de partos gemelares (Tabela 4). Este fato pode ser observado em vários trabalhos com cordeiros de diferentes grupos genéticos (ROCHA et al., 2009; MOHAMMADI et al., 2010; KORITIAKI et al., 2012). A principal razão para que os cordeiros gêmeos se apresentarem mais leves no período pré desmame é a competição intrauterina pelos nutrientes (HAFEZ, 1963) e, após o nascimento, pelo leite materno (ROCHA et al., 2009; MOHAMMADI et al., 2010). De acordo com Mexia et al. (2004) embora as ovelhas de parto gemelar apresentem maior produção de leite, esta não chega a ser o dobro da produção de uma ovelha de parto simples e, deste modo, os cordeiros gêmeos consomem, individualmente, menor quantidade de leite.

A idade da ovelha ao parto influenciou apenas o ganho médio diário de peso do nascimento até o desmame, onde as médias foram maiores nos filhos de ovelhas com idade intermediária (4 e 6 dentes) e menores nos cordeiros filhos de ovelhas mais jovens ( 2 dentes) e mais velhas (8 dentes) (Tabela 5). 
Tabela 5. Médias e erros-padrão do peso, perímetro torácico, comprimento e altura ao nascimento e ao desmame e ganhos médios diários do nascimento ao desmame dessas características de acordo com a idade da mãe ao parto.

\begin{tabular}{|c|c|c|c|c|}
\hline \multirow{2}{*}{ Variáveis } & \multicolumn{4}{|c|}{ Idade da Mãe ao Parto ( $\mathrm{n}^{\circ}$ de dentes incisivos permanentes) } \\
\hline & 2 & 4 & 6 & 8 \\
\hline Peso ao nascimento, $\mathrm{kg}$ & $3,39 \pm 0,34$ & $3,46 \pm 0,26$ & $3,71 \pm 0,19$ & $3,85 \pm 0,19$ \\
\hline Peso ao desmame, $\mathrm{kg}$ & $11,14 \pm 1,28$ & $12,67 \pm 1,01$ & $13,36 \pm 0,71$ & $11,18 \pm 0,72$ \\
\hline Ganho médio diário de peso, $\mathrm{kg}$ & $0,111 \pm 0,017^{\mathrm{ab}}$ & $0,132 \pm 0,013^{\mathrm{a}}$ & $0,138 \pm 0,010^{\mathrm{a}}$ & $0,105 \pm 0,010^{\mathrm{b}}$ \\
\hline Perímetro Torácico ao nascimento, $\mathrm{cm}$ & $36,98 \pm 1,43$ & $36,07 \pm 1,13$ & $38,44 \pm 0,80$ & $37,65 \pm 0,80$ \\
\hline Perímetro Torácico ao desmame, $\mathrm{cm}$ & $55,99 \pm 1,82$ & $54,69 \pm 1,43$ & $55,93 \pm 1,01$ & $53,51 \pm 1,02$ \\
\hline Ganho médio diário de perímetro, $\mathrm{cm}$ & $0,272 \pm 0,029$ & $0,266 \pm 0,023$ & $0,250 \pm 0,016$ & $0,227 \pm 0,016$ \\
\hline Comprimento ao nascimento, $\mathrm{cm}$ & $27,14 \pm 0,84$ & $26,35 \pm 0,67$ & $26,42 \pm 0,47$ & $27,03 \pm 0,47$ \\
\hline Comprimento ao desmame, $\mathrm{cm}$ & $41,39 \pm 1,48$ & $44,57 \pm 1,16$ & $42,54 \pm 0,82$ & $45,52 \pm 0,83$ \\
\hline Ganho médio diário de comprimento, $\mathrm{cm}$ & $0,204 \pm 0,024$ & $0,260 \pm 0,019$ & $0,230 \pm 0,013$ & $0,221 \pm 0,013$ \\
\hline Altura ao nascimento, $\mathrm{cm}$ & $39,64 \pm 1,12$ & $39,62 \pm 0,88$ & $39,30 \pm 0,62$ & $39,25 \pm 0,63$ \\
\hline Altura ao desmame, $\mathrm{cm}$ & $50,91 \pm 2,08$ & $54,84 \pm 1,63$ & $55,21 \pm 1,15$ & $54,66 \pm 1,17$ \\
\hline Ganho médio diário de altura, $\mathrm{cm}$ & $0,161 \pm 0,028$ & $0,218 \pm 0,022$ & $0,227 \pm 0,016$ & $0,220 \pm 0,016$ \\
\hline
\end{tabular}

$\mathrm{a}, \mathrm{b}=$ médias seguidas de letras diferentes na coluna diferem significativamente $(\mathrm{P}<0,10)$ entre si.

Fonte: Elaboração dos autores.

De acordo com Ribeiro et al. (2008b), as borregas mais jovens ainda encontram-se em fase de desenvolvimento e devido a esse fato provavelmente produzem uma menor quantidade de leite, o que explicaria o menor ganho apresentado pelas crias de fêmeas de 2 dentes.

Koritiaki et al. (2012) encontraram menores pesos ao nascer e ao desmame para cordeiros nascidos de ovelhas de 2 dentes, fato não observado neste experimento.
Tanto ao nascimento como ao desmame, as correlações entre os pesos e as medidas corporais foram significativas $(\mathrm{P}<0,05)$ e positivas (Tabela 6). Os coeficientes de correlação encontrados indicam altas correlações entre estas características, sendo o perímetro torácico a medida que melhor se correlacionou com o peso, tanto ao nascimento $(\mathrm{r}=$ $0,68)$ como ao desmame $(r=0,86)$. Estes resultados estão de acordo com os obtidos por Fajemilehin e Salako (2008) e Koritiakiet al. (2012), em estudos com pequenos ruminantes.

Tabela 6. Correlações entre pesos e medidas corporais de cordeiros do nascimento ao desmame.

\begin{tabular}{ccccccccc}
\hline & P0 & P70 & C0 & C70 & PT0 & PT70 & A0 & A70 \\
\hline P70 & $0,64^{* *}$ & - & - & - & - & - & - & - \\
C0 & $0,60^{* *}$ & $0,32^{*}$ & - & - & - & - & - & - \\
C70 & $0,65^{* *}$ & $0,85^{* *}$ & $0,37^{*}$ & - & - & - & - & - \\
PT0 & $0,68^{* *}$ & $0,40^{* *}$ & $0,45^{* *}$ & $0,38^{*}$ & - & - & - & - \\
PT70 & $0,62^{* *}$ & $0,86^{* *}$ & $0,33^{*}$ & $0,81^{* *}$ & $0,45^{* *}$ & - & - & - \\
A0 & $0,68^{* *}$ & $0,52^{* *}$ & $0,61^{* *}$ & $0,47^{* *}$ & $0,50^{* *}$ & $0,57^{* *}$ & - & - \\
A70 & $0,55^{* *}$ & $0,77^{* *}$ & $0,32^{*}$ & $0,78^{* *}$ & $0,33^{*}$ & $0,74^{* *}$ & $0,52^{* *}$ & - \\
GMPD & $0,49^{* *}$ & $0,98^{* *}$ & $0,23^{\mathrm{NS}}$ & $0,81^{* *}$ & $0,29^{\mathrm{NS}}$ & $0,83^{* *}$ & $0,43^{* *}$ & $0.74^{* *}$ \\
\hline
\end{tabular}

$\mathrm{P} 0=$ peso ao nascimento; $\mathrm{P} 70=$ peso ao desmame; $\mathrm{C} 0=$ comprimento corporal ao nascimento; $\mathrm{C} 70=$ comprimento corporal ao desmame; $\mathrm{PT} 0=$ perímetro torácico ao nascimento; $\mathrm{PT} 70=$ perímetro torácico ao desmame; $\mathrm{A} 0=$ altura de cernelha ao nascimento e $\mathrm{A} 70=$ altura de cernelha ao desmame.

** $\mathrm{P}<0,01, * \mathrm{P}<0,05 \mathrm{e}^{\mathrm{NS}}$ não significativo.

Fonte: Elaboração dos autores. 
Ao nascimento, o peso e altura de cernelha dos cordeiros apresentaram correlações moderadas $(\mathrm{r}=$ 0,$49 ; 0,43$, respectivamente) com o ganho médio diário de peso, enquanto que o comprimento corporal e o perímetro torácico não foram correlacionados $(\mathrm{P}>0,05)$ com aquele parâmetro. Entretanto, o ganho médio diário de peso apresentou altas correlações com o peso $(\mathrm{r}=0,98)$, comprimento $(\mathrm{r}=0,81)$, perímetro torácico $(\mathrm{r}=0,83)$ e altura de cernelha $(\mathrm{r}$ $=0,74)$ ao desmame.

\section{Conclusões}

É importante considerar o efeito dos níveis de energia da nutrição da ovelha, pois os mesmos influenciam o desenvolvimento dos cordeiros, podendo afetar a eficiência do sistema de produção. Além da nutrição da ovelha é importante considerar outros fatores ambientais que afetam as características de crescimento dos animais por atuarem na expressão do seu potencial genético. Medidas corporais podem ser utilizadas como indicadores de desempenho dos animais.

\section{Agradecimentos}

Os autores agradecem ao Conselho Nacional de Pesquisa e Desenvolvimento (CNPq) e a Fundação Araucária pelo suporte financeiro.

\section{Referências}

BARROS, N. N.; VASCONCELOS, V. R.; LOBO, R. N. B. Características de crescimento de cordeiros F1 para abate no semi-árido do Nordeste do Brasil. Pesquisa Agropecuária Brasileira, Brasília, v. 39, n. 8, p. 809-814, 2004.

CARNEIRO, P. L. S.; MALHADO, C. H. M.; SOUZA JÚNIOR, A. A. O.; SILVA, A. G. S.; SANTOS, F. N.; SANTOS, P. F.; PAIVA, S.R. Desenvolvimento ponderal e diversidade fenotípica entre cruzamentos de ovinos Dorper com raças locais. Pesquisa Agropecuária Brasileira, Brasília, v. 42, n. 7, p. 991-998, 2007.

CARVAlHO, S.; PIRES, C. C.; PERES, J. R. R.; ZEPPENFELD, C.; WEISS, A. Desempenho de cordeiros machos inteiros, machos castrados e fêmeas, alimentados em confinamento. Ciência Rural, Santa Maria, v. 29, n. 1, p. 129-133, 1999.

CASTRO, F. A. B.; RIBEIRO, E. L. A.; MIZUBUTI, I. Y.; SILVA, L. D. F.; BARBOSA, M. A. A. F.; SOUSA, C. L.; PAIVA, F. H. P.; KORITIAKI, N. A. Influence of pre and postnatal energy restriction on the productive performance of ewes and lambs. Revista Brasileira de Zootecnia, Viçosa, v. 41, n. 4, p. 951-958, 2012.

CAVIGLIONE, J. H.; KIIHL, L. R. B.; CARAMORI, P. H.; OLIVEIRA, D. Cartas climáticas do Paraná. 2000. Disponível em: <http://www.iapar.br/modules/conteudo/ conteudo.php? conteudo=677>. Acesso em: 19 nov. 2012.

FAJEMILEHIN, O. K. S.; SALAKO, A. E. Body measurement characteristics of the West African Dwarf (WAD) Goat in deciduous forest zone of Southwestern Nigeria. African Journal of Biotechnology, Lagos, v. 7, n. 14, p. 2521-2526, 2008.

FERNANDES, A. A. O.; BUCHANAN, D.; SELAIVEVILLARROEL, A. B. Avaliação dos fatores ambientais no desenvolvimento corporal de cordeiros deslanados da raça Morada Nova. Revista Brasileira de Zootecnia, Viçosa, v. 30, n. 5, p. 1460-1465, 2001.

FERNANDES, M. A. M.; MONTEIRO, A. L. G.; BARROS, C. S.; GAZDA, T. L.; PIAZZETTA, R. G.; DITTRICH, J. R.; GASPERIN, C. Desempenho de cordeiros puros e cruzados Suffolk e Santa Inês. Revista da FZVA, Uruguaiana, v. 14, n. 2, p. 207-216. 2007.

GERASEEV, L. C.; PEREZ, J. R. O.; CARVALHO, P. A.; OLIVEIRA, R. P.; QUINTÃO, F. A.; LIMA, A. L. Efeitos das restrições pré e pós-natal sobre o crescimento e o desempenho de cordeiros Santa Inês do nascimento ao desmame. Revista Brasileira de Zootecnia, Viçosa, v. 35, n. 1, p. 245-251, 2006.

GREENWOOD, P. L.; HUNT, A. S.; HERMANSON, J. W.; BELL, A. W. Effects of birth weight and postnatal nutrition on neonatal sheep: II. Skeletal muscle growth and development. Journal of Animal Science, Champaign, v. 78, n. 1 , p. $50-61,2000$.

HAFEZ, E. S. E. Symposium on growth: physio-genetics of prenatal and postnatal growth. Journal of Animal Science, Champaign, v. 22, n. 3, p. 779-791, 1963.

KORITIAKI, N. A.; RIBEIRO, E. L. A.; SCERBO, D. C.; MIZUBUTI, I. Y.; SILVA, L. D. F.; BARBOSA, M. A. A. F.; SOUZA, C. L.; PAIVA, F. H. P. Fatores que afetam o desempenho de cordeiros Santa Inês puros e cruzados do nascimento ao desmame. Revista Brasileira de Saúde e Produção Animal, Salvador, v. 13, n. 1, p. 258-270, 2012. 
MARTIN, G. B.; RODGER, J.; BLACHE, D. Nutritional and environmental effects on reproduction in small ruminants. Reproduction, Fertility and Development, Collingwood, v. 16, n. 4, p. 491-501, 2004.

McDOWELL, L. R.; CONRAD, J. H.; THOMAS, J. E.; HARRIS, L. E. Latin American tables of feed composition. Gainesville: University of Florida, 1974. $509 \mathrm{p}$.

MEXIA, A. A.; MACEDO, F. A. F.; ALCALDE, C. R.; SAKAGUTI, E. S.; MARTINS, E. N.; ZUNDT, M.; YAMAMOTO, S. M.; MACEDO, R. M. G. Desempenhos reprodutivo e produtivos de ovelhas Santa Inês suplementadas em diferentes fases da gestação. Revista Brasileira de Zootecnia, Viçosa, v. 33, n. 3, p. 658-667, 2004.

MOHAMMADI, K.; NASSIRI, M. T. B.; FAYAZI, J.; ROSHANFEKR, $\mathrm{H}$. Investigation of environmental factors influence on pre-weaning growth traits in Zandi lambs. Journal of Animal and Veterinary Advances, Pakistan, v. 9, n. 6, p. 1011-1014, 2010.

NATIONAL RESEARCH COUNCIL - NRC. Nutrient requirement of small ruminants: sheep, goats, cervids and new world camelids. Washington: National Academy Press, 2007. 384 p.

PACHECO, A.; QUIRINO, C. R. Estudo das características de crescimento em ovinos. Pubvet, Londrina, v. 2, n. 29, p. 1982-1263, 2008.

RAE, M. T.; PALASSIO, S.; KYLE, C. E.; BROOKS, A. N.; LEA, R. G.; MILLER, D. W.; RHIND, S. M. Effect of maternal undernutrition during pregnancy on early ovarian development and subsequent follicular development in sheep fetuses. Reproduction, Cambridge, v. 122, p. 915-922, 2001.

RIBEIRO, A. C.; SANTANA, A. F.; LIMA, M. C.; SOUZA, R. S.; NASCIMENTO, J. P. V. Peso ao nascer de ovinos da raça Santa Inês no município de Irará Bahia. PUBVET, Londrina, v. 2, n. 32, p. 1982-1263, 2008a.
RIBEIRO, E. L. A.; MIZUBUTI, I. Y.; SILVA, L. D. F.; ROCHA, M. A.; MORI, R. M. Desempenho produtivo de ovelhas submetidas a acasalamento no verão ou no outono no Norte do Paraná. Semina: Ciências Agrárias, Londrina, v. 29, n. 1, p. 229-236, 2008 b.

ROCHA, L. P.; FRAGA, A. B.; ARAÚJO FILHO, J. T.; FIGUEIRA, R. F.; PACHECO, K. M. G.; SILVA, F. L.; RODRIGUES, D. S. Desempenho de cordeiros cruzados em Alagoas, Brasil. Archivos de Zootecnia, Córdoba, v. 58, n. 221, p. 145-148, 2009.

SANTANA, A. F. de; COSTA, G. B.; FONSECA, L. S. Correlações entre peso e medidas corporais em ovinos jovens da raça Santa Inês. Revista Brasileira de Saúde e Produção Animal, Salvador, v. 1, n. 3, p. 74-77, 2001.

SARMENTO, J. L. R.; TORRES, R. A.; PEREIRA, C. S.; SOUSA, W. H.; LOPES, P. S.; ARAÚJO, C. V.; EUCLYDES, R. F. Avaliação genética de características de crescimento de ovinos Santa Inês utilizando modelos de regressão aleatória. Arquivo Brasileiro de Medicina Veterinária e Zootecnia, Belo Horizonte, v. 58, n. 1, p. 68-77, 2006.

SOUSA, J. E. R.; OLIVEIRA, S. M. P.; LIMA, F. A. M.; SILVA, F. L. R.; SILVA, M. A. Efeitos genéticos e de ambiente para características de crescimento em ovinos Santa Inês no Estado do Ceará. Revista Ciência Agronômica, Fortaleza, v. 37, n. 3, p. 364-368, 2006.

SOUZA, S.; LEAL, A.; BARIONI, C.; MATOS, A.; MORAIS, J.; ARAÚJO, M.; NETO, O.; SANTOS, A.; COSTA, E. R. Utilização de medidas biométricas para estimar peso vivo em ovinos. Archivos Latinoamericanos de Producción Animal, Maracaibo, v. 17, n. 3, p. 61-66, 2009. 